\title{
Wireless Food Monitoring System with High Efficient Long-range Battery-less Smart Sensor Tag
}

\author{
Xuan Tu Cao' ${ }^{1}$, Wan-Young Chung ${ }^{1}$ \\ 1 Department of Electronic Engineering, Pukyong National University, Busan 608-737, South Korea \\ wychung@pknu.ac.kr
}

\begin{abstract}
This paper describes the design of a high efficient long-range battery-less smart sensor Tag using a relay resonator and its applications for meat freshness monitoring. The proposed smart sensor tag using a relay resonator, placed inside the sealed package to measure the emitted gas concentration, relative humidity, and environment temperature for evaluate the quality level of meat. The sensor module is designed using the excellent measurement accuracy sensors at ultra-low power consumption that the total power consumption is less than $1.3 \mathrm{~mW}$. This paper also presents a highly efficient RF energy harvester using a relay resonator which based on resonant coupled wireless power transfer system. An equivalent circuit is used to investigate the effect of relay resonator on harvested power, efficiency and transmission distance. As verified by the analysis and practical measurements, the proposed harvesting method is able to generate approximately 10 times higher power than the conventional energy harvesting methods. By using proposed energy harvesting method, the proposed sensor module can collect the sensing data during the meat spoilages without using any batteries at maximum distance $50 \mathrm{~cm}$. The collected sensing data are analyzed to evaluate the freshness of meat packages.
\end{abstract}

Key words: Smart sensor tag, equivalent carbon dioxide sensor, temperature and humidity sensor, meat freshness monitoring, relay resonator.

\section{Introduction}

Nowadays, meat freshness insurance becomes a critically important issue for the consumer safety. During the food supply chain, the consumer usually evaluates the meat freshness relies on sensory assessment of freshness attributes, such as texture, smell, appearance, and color. However, these methods are inaccurate to predict the potential harmful as well as the meat shelf-life. Therefore, many methods to monitor the food freshness has been proposed (1). These methods are provided sensor devices which use the $\mathrm{H}_{2} \mathrm{~S}$ gas, $\mathrm{CO}_{2}$ gas sensor, relative humidity, temperature sensor... to evaluate the meat freshness during the storage time. Although the sensor, which low cost, compact size, and lowpower consumption, these smart sensor tags still can't operate continuously in fully passive mode. The transmission distance is also limited by the power issues.

In order to overcome the limitation of conventional smart sensor tag, we proposed a new high efficient smart sensor system using a relay resonator. The proposed smart sensor tag consists of a smart sensing module, an energy harvester, and a relay resonator. This proposed tag can measure the sensing data inside the sealed packages during the experiments. The relay resonator has been attached to the straight line-of-sight between a reader and proposed tag. By the analysis and practical power measurements, we demonstrated the effect of relay resonator on the harvested power and transmission distance compare with the conventional system without a relay resonator. With using the relay resonator, the proposed tag doesn't need to use any batteries to supply power during food monitoring experiments in two temperature conditions.

\section{System design and Implementation}

The block diagram of proposed meat freshness monitoring system consists of a reader, a wireless battery-less smart sensor tag, a server and a relay resonator as shown in Fig.1. The development of wireless battery-less smart sensor tag that includes ultra-low power sensor. The transmission range of the system is 
approximately $50 \mathrm{~cm}$ in a free-space environment to proximity meat freshness monitoring continuously in real time. The wireless battery-less smart sensor tag is designed to monitor the temperature and gas sensing during the meat spoilages on two different environments.

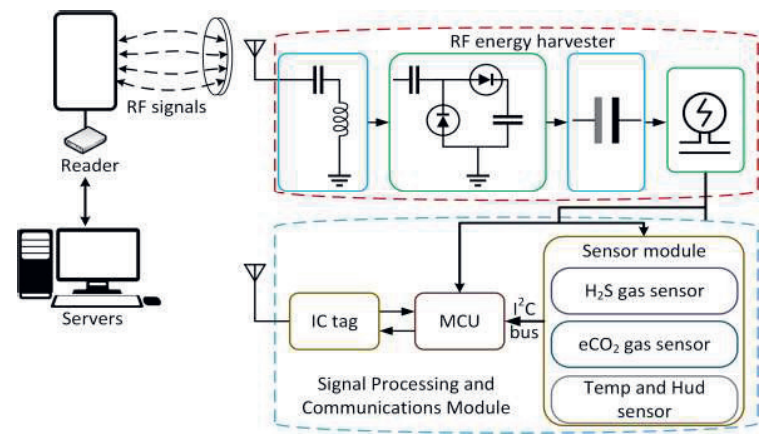

Fig.1.The block diagram of proposed wireless longrange battery-less smart sensor system.

No batteries are required for this proposed sensor tag. In order to overcome this problem, two sensors have been carefully chosen to have ultra-low power consumption during their operation. These sensors (HDC1080 (TI, USA) and CCS811 (AMS, Austria)) has been selected to measure relative humidity, temperature, and eCO2 gas concentration.

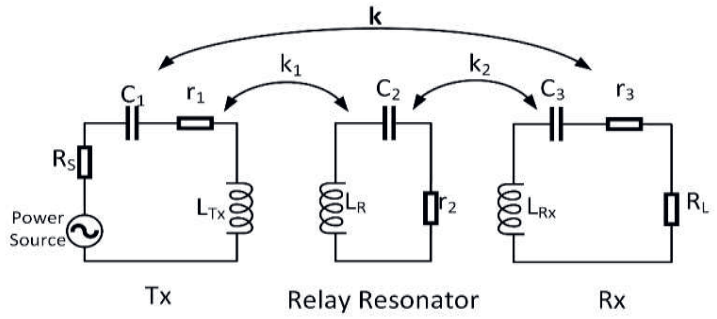

Fig. 2. The block diagram of proposed wireless longrange battery-less smart sensor system.

Most studies recent, they usually use the RF energy harvesting system include the transmitter and receiver to scavenge the energy and supply for whole circuit. However, the harvested power was too small and thereby the system can't operate in full passive mode. Therefore, the proposed energy harvester using a relay resonator has been presented. Solving the full Kirchoff's voltage law (KVL) on the equivalent circuit in Fig.2, we demonstrate the highly efficient energy performance and maximum power transmission. We prepared some samples for the experiment of meat freshness monitoring. The experiments were done 10 times in 8 days. The proposed smart sensor tag is placed inside the package to measure the temperature, relative humidity, and gas concentration. It energized by a 4-watt reader at a distance range $50 \mathrm{~cm}$ away from the smart sensor tag. The PC server was used to illustrate the sensing data, storing and predict the quality of food.

\section{Experiment results and Discussion}

As the analysis and practical measurement, Fig. 3 shows the power measured from reader to the tag using a relay resonator. By using the relay resonator, the harvested power is bigger approximate 10 times than the traditional power transfer system, which scans from 10 to $60 \mathrm{~cm}$.

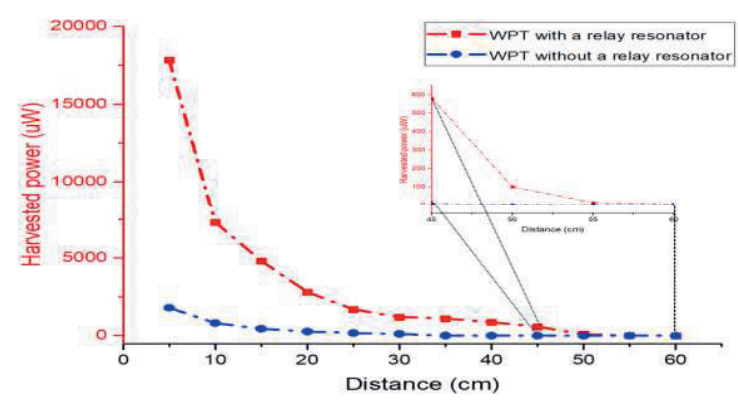

Fig. 1. The changing of harvested power based on the optimum relay resonator position.

Fig. 4 shows the sensing data measured from the proposed sensor tag. In the two difference environment experiments, The $\mathrm{eCO}_{2}$ gas concentration in meat package increase linearly over 8-day tests. There is slow increase in the emitted gas concentrations when the meat package put inside the refrigerator.

In this study, the proposed battery-less smart sensor system has been designed and implemented to monitor the meat freshness. In addition, a highly efficient RF energy harvester using a relay resonator was integrated to supply power for the entire circuit without any batteries at the maximum distance of $50 \mathrm{~cm}$.

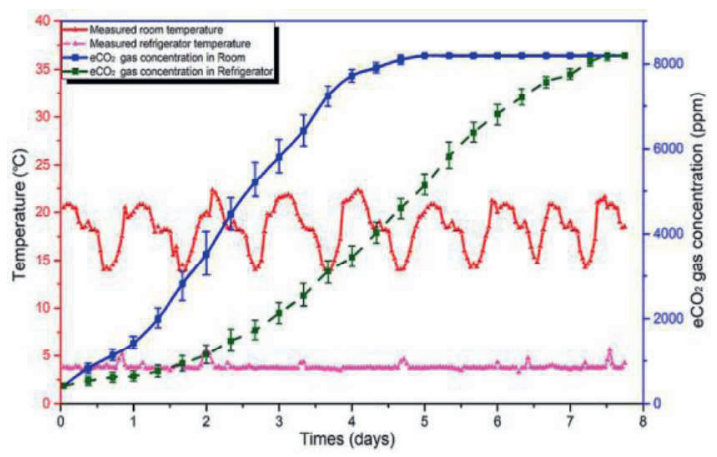

Fig.3. The collected sensing data during 8 days in two temperature environments.

\section{References}

[1] W. Y. Chung, G. T. Le, T. V. Tran, N. H. Nguyen, Novel proximal fish freshness monitoring using batteryless smart sensor tag, Sensor and Actuators B 248, 910-916 (2017).

[2] Dukju Ahn, Songchel Hong, A Study on Magnetic Field Repeater in Wireless Power Transfer, IEEE Transactions on Industrial Electronics 60, 360371 (2013). 